\title{
Desmoplastic non-infantile ganglioglioma: A low-grade tumor, report of two patients
}

\author{
Shaila R. Khubchandani, Arun R. Chitale, Paresh K. Doshi ${ }^{1}$ \\ Departments of Histopathology and ${ }^{1}$ Neurosurgery, Jaslok Hospital and Research Center, BG Deshmukh Marg, Mumbai - 200026 , \\ India
}

Address for correspondence:

Dr. Shaila Khubchandani,

31, Kailas Darshan,

Kennedy Bridge, Nana Chowk,

Mumbai - 400 007, India.

E-mail: shailakhubchandani@

yahoo.co.in

\begin{abstract}
Desmoplastic infantile gangliogliomas (DIGG) are low-grade (WHO Grade I) tumors of early childhood rarely occurring in older children and young adults. The mainstay of treatment is surgical. We report two older children with DIGG, both showing atypical radiology. One case was treated with radiation. The recurrence treated with only surgical excision showed excellent response to treatment.
\end{abstract}

Key words: Desmoplastic non-infantile ganglioglioma, collagen

\section{Introduction}

Desmoplastic ganglioglioma and desmoplastic astrocytoma are infantile tumors and the characteristic trait of these tumors is desmoplasia, imparting firmness to the neoplasms. Desmoplastic infantile ganglioglioma (DIGG) is made up of both astrocytes and neuronal cells. ${ }^{[1]}$ The first report of DIGG, earlier known as desmoplastic supratentorial neuroepithelial tumor, was by Vandenberg and colleagues. ${ }^{[2]}$ We report two cases of DIGG, both occurring in older children and one that recurred after radiation therapy.

\section{Case Report}

\section{Case 1}

A seven-year-old boy presented with enlarging head circumference and skull deformity of four months duration. Neurological examination was essentially normal. The computed tomography (CT) scan revealed a large supratentorial heterogeneous multiloculated cystic and solid mass lesion in the left temporoparietal lobe with well-defined margins and temporal bone scalloping. The cysts were isointense on T1-weighted image (T1-WI) and hyperintense on T2-weighted image (T2-WI). The solid component was hypointense on both T1-WI and T2-WI sequences [Figure 1]. The solid component was peripherally located to the cystic component and revealed intense enhancement with gadolinium. There was thick linear enhancement of the adjacent tentorial leaflet. A left temporal craniotomy was performed and only the solid component of the tumor was excised.

The histological examination revealed a tumor composed of astrocytes and ganglion cells [Figure 2a] interspersed by bands of hyalinization [Figure 3a]. The astrocytes stained with glial fibrillary acidic protein (GFAP) [Figure 2b]. The ganglion cells were positive for neuron specific enolase (NSE) and synaptophysin [Figure 2c]. Masson's stain confirmed the collagen content [Figure 3b]. MIB1 stain (proliferation marker) was negative.

In all Grade I tumors radiotherapy is not indicated. However, in this child therapy in the form of 54 Gy was administered, since only a part of the tumor was removed. A few months later the child presented with seizures which were well controlled with antiepileptic drugs. The patient showed no neurological abnormality and the visual evoked responses were unremarkable. Electroretinogram revealed symmetrical borderline abnormality in the rod system. Repeat imaging revealed a large heterogeneous cystic mass with solid enhancing components in the left temporoparietal region and ventricular trigone. Multiple cysts were seen extending into the suprasellar and cerebellopontine angle cisterns. The crus cerebri was compressed by the cyst in the cerebellopontine 

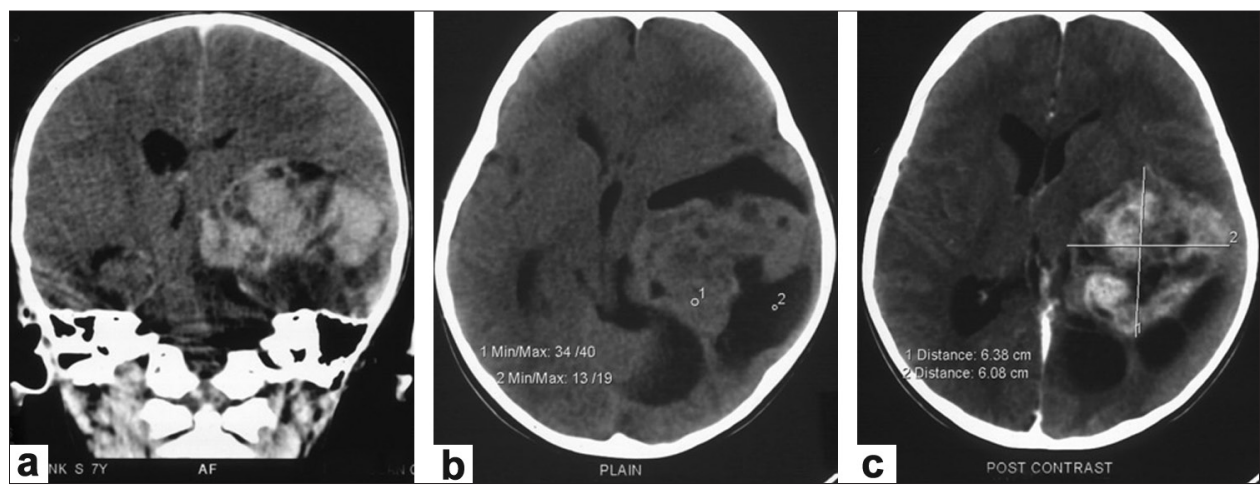

Figure 1: Post-contrast coronal computed tomography; (a) and plain axial; (b) and post-contrast axial; (c) computed tomography scans reveal a left temporoparietal lobe enhancing solid mass with multiple thin-walled cystic components along the periphery of the mass
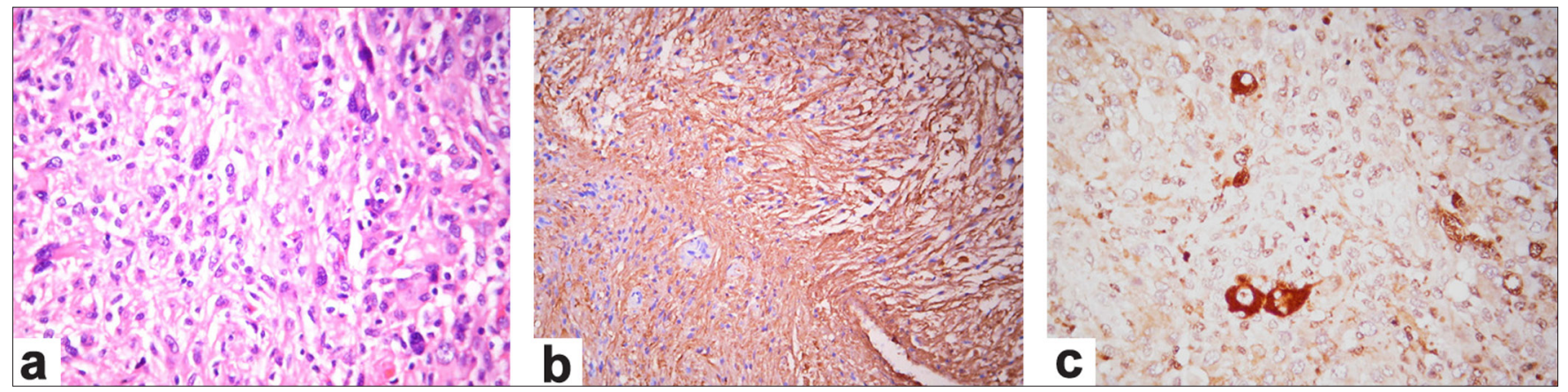

Figure 2: (a) The tumor reveals an admixture of fusiform astrocytes and ganglion cells $(\mathrm{H}$ and E, $\times 40)$; (b) The presence of astrocytes is confirmed with glial fibrillary acidic protein staining (GFAP, $\times 40$ ); (c) The strong cytoplasmic brown stain confirms the neurogenic differentiation (Synaptophysin, $\times 40$ )
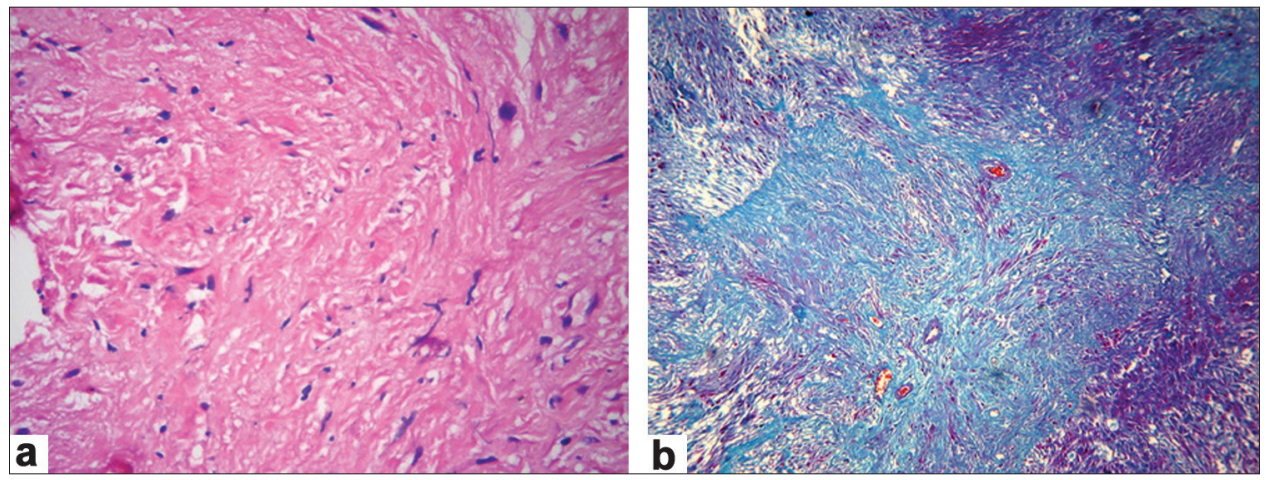

Figure 3: (a) This tumor rich in astrocytes and ganglion cells shows prominent desmoplasia ( $\mathrm{H}$ and E, $\times 40)$; (b) The strong desmoplasia confirmed by the green staining (Masson, $\times 40$ )

cistern [Figure 4]. The patient was re-explored. The solid component was excised and the cysts in the cerebellopontine and suprasellar cisterns were opened and decompressed. A follow-up scan revealed complete excision of the solid component, decompression of the cysts and a widely opened ventricular trigone. The light microscopy showed identical histology of a DIGG.

\section{Case 2}

An eight-year-old boy presented with two episodes of generalized tonic-clonic seizures. Neurological evaluation revealed no abnormality. The CT scan showed a $3.5 \times 2.8 \mathrm{~cm}$ well-defined calcified, solid mass, located in the left peritrigonal region compressing the left occipital horn. Contrast studies revealed minimal enhancement. T1-WI images confirmed a solid mass devoid of cysts or perifocal edema. T1-WI images showed multiple hypointense regions corresponding with the calcified foci. There was intense enhancement of the mass on gadolinium injection [Figure 5]. The mass was completely excised and histology revealed a DIGG confirmed with immunohistochemistry. MIB1 staining was negative. The ultrastructural examination also showed astrocytic [Figure 6a] and ganglionic differentiation [Figure 6b]. Both patients are doing well on follow-up of two years. 


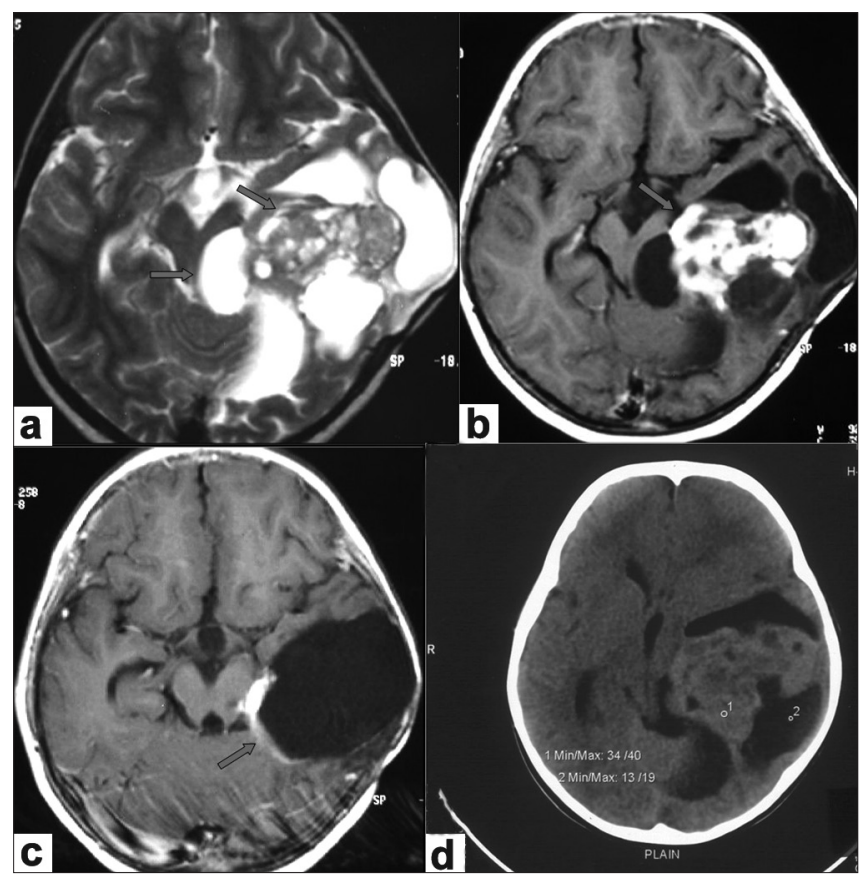

Figure 4: Case 1; Axial T2 (a), axial T1 (b) images, large supratentorial heterogeneous tumor involving temporoparietal lobes with mass effect. Tumor has solid/cystic components. Post-contrast axial T1WI (c) images reveal heterogeneous enhancement of the solid component. postcontrast T1WI axial scan (d) reveals complete removal of the mass with residual cystic cavity in the left temporoparietal lobe

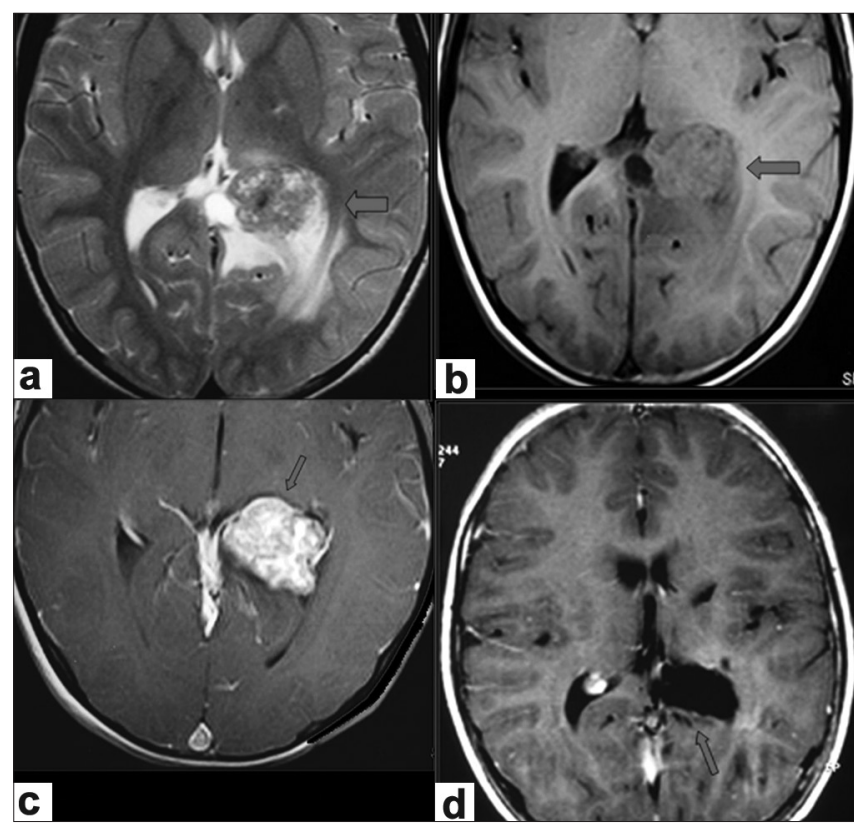

Figure 5: Case 2; Axial T2 (a), and axial T1 (b), images show a solid mass in the left peritrigonal region. Multiple hypointense regions of calcifications are seen within the tumor on both T1WI and T2WI images. Post-contrast T1WI (c), image reveals homogenous dense enhancement of the solid peritrigonal mass. Post-contrast T1WI (d) axial image reveals complete removal of left peritrigonal mass
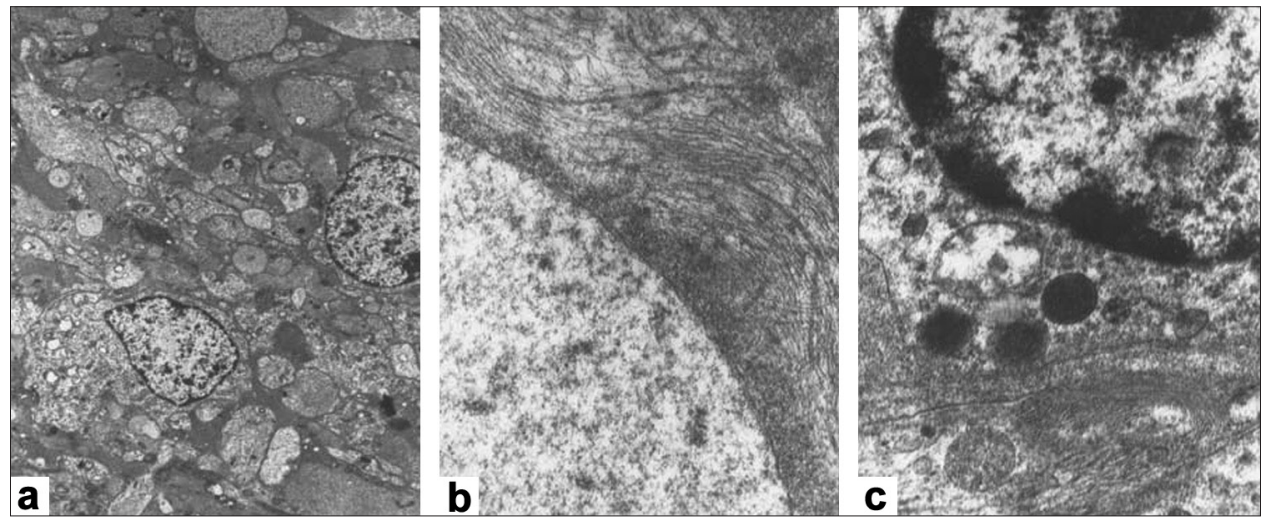

Figure 6: (a) Intertwining cell processes packed with intermediate filaments $(\times 6000)$; (b) Situated near the cells are bundles of collagen $(\times 20000)$; (c) The cells are rich in membrane-bound granules noted in ganglion cells $(\times 20000)$

\section{Discussion}

Desmoplastic infantile ganglioglioma commonly occurs in infants below the age of 18 months, (average four to six months) and mostly described below the age of four years. A few exceptional cases like our patients are recorded in older children and young adults. ${ }^{[3]}$ The tumors are mainly supratentorial, involving multiple lobes with a predilection for the fronto- parietal region. ${ }^{[1]}$ The tumors appear heterogeneous on magnetic resonance imaging (MRI) due to cystic degeneration and collagen in the tumor. The clinical manifestations are mainly those of hydrocephalus and mass lesion. This rare tumor accounts for only $0.04 \%$ of all brain tumors. ${ }^{[1]}$

Desmoplastic infantile gangliogliomas are voluminous, show intense desmoplasia, divergent astrocytic and ganglionic differentiation. Diagnosis can be suspected on MRI and the histological diagnosis is relatively easy in most cases. Rarely, immunohistochemistry is necessary to demonstrate the presence of the astrocytic (with GFAP) and neuronal (with NSE, synaptophysin and neuro filaments) elements. Infrequently, DIGG may be composed of small cells showing mainly neuronal differentiation. Occasionally, these cells are so immature that the neuronal differentiation is ascertained only by the presence of microtubule-associated protein and 
neuron-specific beta tubulin. The ultrastructure reveals astrocytes, fibroblasts and neuronal cells. These tumors arise from the multipotent cells of the neural plate. ${ }^{[4]}$ They are considered as a variant of a ganglioglioma showing differential growth potential. It has been postulated that some tumors may arise in utero as they manifest in very early infancy. The differential diagnosis of DIGG includes primitive neuroectodermal tumor (PNET'), metastatic sarcoma, glioblastoma ${ }^{[5]}$ and gliosarcoma.

The treatment of choice is surgery as these tumors are mostly superficially located and occur in young children before the fontanels fuse. They have an excellent prognosis after excision. ${ }^{[-8]}$ In a few cases, spontaneous regression has been reported, even after subtotal excision. ${ }^{[9]}$ These tumors rarely recur and recurrences have also shown good response to surgical treatment. A few case reports have documented metastatic disease. ${ }^{[10]}$ The role of adjuvant therapy is yet very limited. Chemotherapy is given only to those patients having high-grade tumors showing brisk mitosis, aneuploidy and increased MIB labeling. It is also given to patients with tumors involving eloquent regions of the brain not amenable for surgery ${ }^{[5]}$ Majority of the tumors do not demonstrate any genetic instability. In most situations, as seen in Case 1 radiotherapy has no role to play in the management of DIGG. ${ }^{[6]}$

\section{Conclusion}

This rare Grade I (World Health Organization) voluminous superficial tumor having specific radiological and histological features, reported in the past mainly in early infancy may also be seen in older children. The mainstay of treatment in the majority of cases is only surgical with excellent prognosis.

\section{References}

1. VandenBerg SR. Desmoplastic infantile gangliogliomas and desmoplastic cerebral astrocytomas of infancy. Brain Pathol 1993;3:275-81.

2. VandenBerg SR, May EE, Rubinstein LJ, Herman MM, Perentes E, Vinores SA, et al. Desmoplastic supratentorial neuroepithelial tumors of infancy with divergent differentiation potential ("desmoplastic infantile gangliogliomas"): Report of 11 cases of a distinctive embryonal tumor with favorable prognosis. J Neurosurg 1987;66:58-71.

3. Marti A, Almostarchid B, Maher M, Saidi A. Desmoplastic non-infantile ganglioglioma: Case report. J Neurosurg Sci 2000;44:150-4.

4. Komori T, Scheithauer BW, Parisi JE, Watterson J, Priest JR. Mixed conventional and desmoplastic infantile ganglioglioma: An autopsied case with 6-year follow-up. Mod Pathol 2001;14:720-6.

5. Tamburrini G, Colosimo C Jr, Gaingaspero F, Riccardi R, Di Roceo C. Desmoplastic infantile gangliogliomas. Childs Nerv Syst 2003;19:292-7.

6. Bachli H, Avoledo P, Gratzl O, Tolnay M. Therapeutic strategies and management of desmoplastic infantile gangliogliomas: Two case reports and literature overview. Childs Nerv Syst 2003;19:359-66.

7. Alexiou GA, Stefanaki K, Sfankianos G, Prodromou N. Desmoplastic infantile ganglioglioma: A report of 2 cases and a review of the literature. J Neurosurg Pediatr 2008;1:95-8.

8. Lonnort K, Terho M, Kahara V, Haapasalo H, Helén P. Desmoplastic infantile ganglioglioma: Novel aspects in clinical presentation and genetics. Surg Neurol 2007;68:304-8.

9. Takeshima H, Kawahara Y, Hirano H, Obara S, Niiro M, Kuratsu J. Postoperative regression of desmoplastic infantile gangliogliomas report of two cases. Neurosurgery 2003;53:979-84.

10. Darwish B, Arbuckle S, Kellie S, Besser M, Chaseling R. Desmoplastic infantile ganglioglioma/astrocytoma with cerebrospinal metastasis. J Clin Neurosci 2007;14:498-501.

Accepted on 04-02-2009

Source of Support: Nil, Conflict of Interest: None declared. 